\title{
AUTHORS INDEX
}

Acosta-Lomelí, M.E.

Aguilar, M.

Almeraya-Calderón, F.

Alvarado-Gámez, A.L.

Arnanz, A.

Arteaga, C.C.

Avaca, L.A.

Banczek, E.P.

Basha, C.A.

Berd, $\mathrm{M}$.

Campos-Fernández, J.

Carmo, D.R.

Chavarín, J.U.

Codognoto, L.

Costa, J.M.C.

Cunha, M.T.

D'Elia, L.F.

Delgado, S.

Domínguez-Sánchez, G.

Dubey, A.

Durstewitz, C.B.

Espinoza-Castellón, E.R.

Fouda, A.S.

Gaus, E.

Hammadi, A.

Herrera, M.T.A.

Jaquez, R.N.

Kalaiselvi, N.

Kulandainathan, M.A.

Kulangiappar, K.

León-Rojas, C.

Lobo, V.M.M.

Machado, S.A.S.

Mahmoud, S.S.

Marcos, M.L.

Martínez, G.

Martinez, M.A.

Miranda, M.L.

Monika

Moreno, M.C.

Mossi, A.C.

Mostafa, H.A.

Moussa, M.N.

Muniyandi, N.

Muthukumaran, A.

Nagiub, A.M.

Núñez-Jaquez, R.E.

Oliveira, M.F.
179,413

77

$17,47,111,123,179,413$

207, 393

35

3

225

379

367

437

207

457

3

225

289

379

481

35

47

445

111,123

393

275

471

437

17

123

247

355

355

$139,195,333,393$

223

225

423

35

55

3

195

445

35

89

275

275

247

355

301

111

379 
Ortíz, R. 481

Pariona, M.M. $\quad 89$

Pereira, C.M. 263

Periasamy, P. 247

Quirós, M.B. 165, 403

Raju, T. $\quad 355,367$

Rastogi, R.B. $\quad 315$

Reyna, J.M. $\quad 77$

Rios, G.B. $\quad 17$

Rodrigues, P.M.S. $\quad 289$

Rodrigues, P.R.P. $\quad 379$

Roquero, P. $\quad 77$

Sádaba, M. $\quad 55$

Sakthivel, M. 247

Sánchez, M. $\quad 55$

Santos, H.A. 263

Siddique, W.A. $\quad 445$

Silva, F. 263

Silva, R.M. $\quad 457$

Singh, K. $\quad 315$

Singh, M.M. $\quad 315$

Staroin, F.O.R. $\quad 89$

Stradiotto, N.R. $\quad 457$

Thirunakaran, R. 247

Tiburcio, C.G. 47, 111, 123, 179, 413

Torrent-Burgués, J. 471

Uruchurtu-Chavarin, J. 111

Valente, A.J.M. 223

Velasco, J.G. 35

Velásquez-González, R. $\quad 179,413$

Villafañe, A.M. $\quad 47,123,111,179,413$

Wu, B.Z. 165

Yadav, M. $\quad 315$ 